CARNETS OE Carnets de géographes

GÉOGRAPHES.

$1 \mid 2010$

Varia

\title{
Regard et place d'une chercheuse blanche dans les espaces publics de Johannesburg
}

Pauline Guinard

\section{(2) OpenEdition \\ Journals}

Édition électronique

URL : http://journals.openedition.org/cdg/1911

DOI : 10.4000/cdg. 1911

ISSN : 2107-7266

Éditeur

UMR 245 - CESSMA

Référence électronique

Pauline Guinard, «Regard et place d'une chercheuse blanche dans les espaces publics de

Johannesburg », Carnets de géographes [En ligne], 1 | 2010, mis en ligne le 01 octobre 2010, consulté le 07 mai 2019. URL : http://journals.openedition.org/cdg/1911 ; DOI : 10.4000/cdg.1911

La revue Carnets de géographes est mise à disposition selon les termes de la Licence Creative Commons Attribution - Pas d'Utilisation Commerciale - Pas de Modification 4.0 International. 


\title{
REGARD ET PLACE D'UNE CHERCHEUSE BLANCHE DANS LES ESPACES PUBLICS DE JOHANNESBURG
}

\author{
Pauline Guinard \\ pauline.guinard@gmail.com \\ Doctorante-enseignante contractuelle sous la direction de Philippe Gervais-Lambony \\ Laboratoire Gecko (EA 375), Ecole Doctorale Milieux, cultures et sociétés du passé et du présent \\ Université Paris Ouest Nanterre
}

Notes de l'auteur. Les recherches de terrain, dont rend compte cet article, ont été réalisées grâce au soutien de l'IFAS (Institut français d'Afrique du Sud) et de l'école d'architecture et d'aménagement de l'Université du Witwatersrand (Johannesburg, Afrique du Sud).

S'intéresser aux espaces publics suppose tout d'abord de pouvoir cerner son objet d'étude. Or, la notion même d'espace public pose problème, étant donné la multiplicité des définitions qu'elle recouvre (Paquot, 2010). De manière générale, on peut en distinguer trois sens : un sens juridique - les espaces publics comme espaces non-détenus par des propriétaires privés; un sens social - en tant qu'espaces de rencontre potentielle avec l'altérité ; et un sens politique - comme espaces de débat. Ces trois dimensions ne correspondent pas nécessairement aux mêmes espaces. Au contraire, dans le contexte contemporain de la globalisation, ces trois types d'espace, et notamment les espaces juridiquement et socialement publics, auraient tendance à se dissocier géographiquement (Ghorra-Gobin, 2001). Ainsi, les espaces juridiquement publics tels que la rue, les places ou les parcs, ne correspondraient plus ni aux espaces de débat public ni surtout aux espaces d'interactions sociales, alors que les espaces privés, à l'image des centres commerciaux, deviendraient des espaces socialement publics par les pratiques qui s'y font jour (Capron, 1998 ; Houssay-Holzschuch, Teppo, 2009).

Cette tendance se retrouve à Johannesburg où les espaces juridiquement publics sont aujourd'hui soumis à des logiques de privatisation et de sécurisation qui remettent en cause leur caractère public. Ce processus y est d'autant plus accentué que ces espaces sont dangereux, ou du moins perçus comme tels ${ }^{1}$ par la plupart des usagers de la ville, et plus particulièrement par la population blanche et/ou riche. Le statut des espaces publics à

\footnotetext{
${ }^{1}$ Malgré la difficulté à obtenir des chiffres fiables, les taux d'homicides volontaires et de viol en Afrique du Sud, estimés respectivement à 37,6 pour 100000 personnes en 2008 et 117 pour 100000 personnes en 2002 par l'office des Nations Unies sur les drogues et le crime (United Nations Office on Drugs and Crime -UNODC) sont considérés comme faisant partie des plus élevés au monde. Par ailleurs, en dépit d'une relative stabilité de ces taux à Johannesburg depuis 1994, la perception de la violence devient de plus en plus aiguë (Boisteau, 2003).
} 
Johannesburg est en effet encore complexifié par les héritages de la ségrégation, puisque ces espaces ont longtemps été divisés selon un critère « racial », et ceci même avant l'instauration de l'apartheid (1948-1991) ${ }^{2}$. Cette ségrégation tendait à nier la notion même d'espace public dans toutes ses dimensions (Houssay-Holzschuch, Teppo, 2009), en empêchant notamment la constitution d'espaces de rencontre entre les différentes catégories " raciales ». Dès lors, étudier les espaces juridiquement publics de Johannesburg pour en interroger la dimension sociale suppose, en particulier pour une femme blanche, un certain nombre de contraintes méthodologiques qui forment et informent l'objet de recherche.

Mon but n'est pas ici de revenir sur les débats théoriques autour de ce que sont les espaces publics, mais de comprendre en quoi l'expérience de terrain permet de mieux saisir la réalité de ces espaces à Johannesburg. Ainsi, en étudiant les espaces juridiquement publics au prisme de l'art installé dans ces espaces, j'entends m'interroger sur leur caractère socialement voire politiquement public à travers l'analyse des pratiques des usagers. Mais, de même que ces pratiques façonnent les espaces publics, elles influencent les méthodes du chercheur. Dès lors, en quoi la pratique des espaces publics johannesburgeois me permet-elle ou m'empêchet-elle - en tant que chercheuse blanche - de cerner ce que sont ces espaces ?

\section{À la recherche des espaces publics...}

Comme je l'ai mentionné précédemment, les espaces juridiquement publics de Johannesburg sont des espaces évités. Cela résulte à la fois de l'héritage ségrégatif de l'apartheid et de la perception, fondée ou non, de ces espaces comme dangereux. Ainsi, les personnes présentes dans les espaces publics ne s'y trouvent que très rarement par choix. Ce sont des personnes pauvres, bien souvent noires qui, n'ayant pas de voiture, y attendent le bus ou les taxis collectifs ${ }^{3}$. Observer les passants dans les espaces publics sud-africains induit un biais majeur en termes de représentativité, puisque les personnes étudiées sont principalement des populations pauvres et noires. Faut-il alors chercher le public blanc et riche hors des espaces juridiquement publics ? Si oui, qu'est-ce que cela implique quant à la définition des espaces publics? Les espaces juridiquement publics ne seraient-ils que des espaces de relégation pour des populations qui n'ont pas les moyens de les éviter ? Pourraient-ils alors être encore considérés comme socialement publics?

Les photographies choisies par la municipalité pour donner un aperçu de la ville sur son site Internet sont révélatrices de ce que sont la rue et les places à Johannesburg. Lorsqu'ils sont représentés, ces espaces sont quasiment vides d'hommes, quoique parcourus par des flots plus ou moins importants de voitures. Ceci vaut particulièrement pour le centre-ville, ancien cœur économique de la ville entré dans un processus de déclin depuis les années 1970 (Beavon, 2004), et aujourd'hui réputé dangereux :

\footnotetext{
${ }^{2}$ En 1950, le gouvernement d'apartheid vote le Population Registration Act, loi qui divise la population en quatre groupes « raciaux » (Blanc, Noir, Coloured, Indien) selon la terminologie alors employée par le régime. Bien que cette loi marque un tournant, les politiques ségrégatives se mettent en place en Afrique du Sud, et à Johannesburg, dès le début du XX ${ }^{\text {ème }}$ siècle (Guillaume, 2001).

${ }^{3}$ Les taxis collectifs sont des sortes de minibus dont le parcours est plus ou moins prédéterminé, et qui permettent de suppléer au manque de transports en commun pour les populations les plus modestes.
} 


\section{Les espaces juridiquement publics du centre-ville de Johannesburg, des espaces sans} public ?

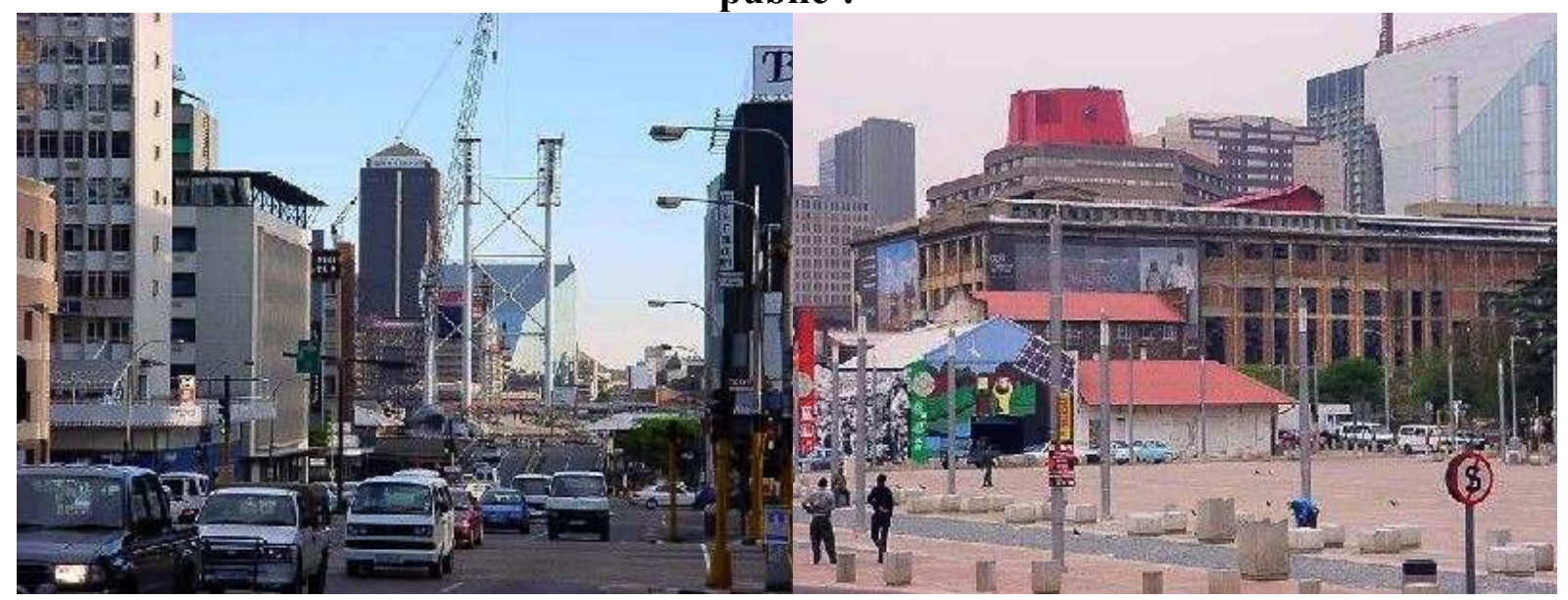

(C) Reproduit avec l'autorisation du site Internet “City of Johannesburg”, http://www.joburg.org.za

D'après ces photographies, les espaces juridiquement publics semblent essentiellement pratiqués en voiture, et non à pied. Les automobilistes font-ils partie du public des espaces publics? Si tel est le cas, cela implique de réviser non seulement la définition de ces espaces, mais aussi de s'interroger sur la nature des liens interpersonnels possibles dans un tel contexte. On s'approcherait en effet d'un modèle de citoyenneté du type «S.U.V » ${ }^{4}$ (Sports Utility Vehicle ou véhicule utilitaire sportif) tel que défini par D. Mitchell (Mitchell, 2005), c'est-à-dire une forme de citoyenneté dans laquelle le public - ou du moins la partie du public qui pourrait se le permettre - ne serait qu'une somme d'individus atomisés qui, au nom de leur liberté et de leur sécurité, s'enfermeraient dans des sortes de bulles, au sens propre ou au figuré, plus ou moins infranchissables. La voiture serait une bulle de ce type rendant possible un déplacement dans les espaces publics juridiques, mais préservant l'usager de toute rencontre fortuite, tout comme le centre commercial serait une autre bulle permettant un contact avec autrui dans un espace public social contrôlé. Dès lors, étudier les espaces publics en étant dans ces espaces a-t-il un sens?

\section{Quelle place pour une femme blanche dans les espaces publics johannesburgeois?}

Choisir d'étudier les espaces publics en étant dans les espaces publics relève d'un choix méthodologique, pas nécessairement conscient au départ, qui postule, selon le modèle européen, que les espaces publics se parcourent à pied. Or, du caractère partiellement public de ces espaces découle toute une série d'obstacles qui invite le chercheur à interroger et repenser ses techniques d'enquête, en l'occurrence observation et questionnaires ${ }^{5}, \mathrm{~s}^{\prime}$ il ne veut pas passer à côté de son objet.

Tout d'abord, la rareté des bancs ou des cafés donnant sur la rue qui permettraient de contempler l'espace environnant est un premier frein pour l'observation, et confirme une certaine conception des espaces publics comme espaces de passage et de circulation, et non

\footnotetext{
${ }^{4}$ Les S.U.V. sont des véhicules surélevés, très répandus aux Etats-Unis et au Canada, et de plus en plus en Europe où ils sont généralement qualifiés de $4 \mathrm{X} 4$.

${ }^{5}$ Bien que cette question soit cruciale, notamment pour interpréter les résultats de mes enquêtes, je ne détaillerai pas ici le type de questionnaires utilisés, car mon propos est pour le moment de m'interroger sur les implications de mon rapport aux usagers des espaces publics de Johannesburg, ce rapport étant considéré préalablement à tout questionnaire.
} 
d'accueil et de rencontre. Ensuite, des comportements tels que s'arrêter ou marcher dans les espaces publics peut apparaître suspect pour les passants, surtout quand on est une femme blanche. Ainsi, lors de mon premier terrain à Johannesburg en février 2009, alors que je traversais le centre-ville en pleine journée, une femme noire d'une cinquantaine d'années m'a dit: «Ce n'est pas une rue pour Blancs. Garde ça en tête, chérie ! (It's not a street for White. Keep it in mind, baby!). Cette femme cherchait sans doute à m'informer, à me protéger plutôt qu'à m'intimider. Mais la manière dont elle s'est exprimée est significative. Elle ne m'a pas dit que c'était un quartier dangereux, mais que ce n'était pas un quartier pour les Blancs, sous-entendant vraisemblablement, à l'image des discours dominants en Afrique du Sud : c'est un quartier noir (pauvre) et donc dangereux pour les Blancs (riches). Par ailleurs, le besoin qu'a ressenti cette femme de m'avertir ne tenait probablement pas seulement à ma couleur, car premièrement, dans la société sud-africaine marquée par des taux records de viol, le fait que je sois une femme seule dans l'espace public me met dans une position de victime potentielle, ce qui a sans doute motivé le désir de protection de cette femme; et deuxièmement, cette femme m'a immédiatement perçue comme étrangère. En effet, contrairement à ce qui peut se passer dans d'autres pays d'Afrique sub-saharienne, être blanche à Johannesburg ne signifie pas forcément être étrangère. Par contre, le fait d'être blanche et de se trouver dans cet espace indiquait clairement que je n'étais pas de Johannesburg, puisque je ne savais pas que les Blancs en Afrique du Sud ne fréquentent pas les espaces publics, surtout ceux du centre-ville (Mabin, 2001). Mon comportement ne répondant pas aux normes implicites de l'espace parcouru, j'étais « out-of-place » (Cresswell, 1996), hors du lieu. Involontairement, ma présence était perçue par les autres usagers de l'espace, et en l'occurrence par cette femme noire, comme une transgression, voire comme une agression. Au final, cet incident est symptomatique du caractère encore ségrégué et " gendré » des espaces publics johannesburgeois. Le fait d'être blanche et d'être une femme à Johannesburg détermine malgré moi dans le regard des autres les espaces auxquels j'ai accès ou non.

\section{Trouver ou refuser une place dans les espaces publics : deux études de cas}

Etre une femme blanche dans les espaces publics à Johannesburg apparaît comme une double contrainte méthodologique, liée à la couleur et au genre. D'une part, la présence d'un chercheur blanc dans les espaces publics du centre-ville peut être vécue comme surprenante, et même provocatrice vis-à-vis des autres usagers, majoritairement noirs et pauvres. Les motifs de ma présence en ces lieux qu'eux-mêmes préféreraient ne pas fréquenter leur sont obscurs. Le chercheur, par le regard d'autrui, est ainsi amené à s'interroger sur le sens et la portée de sa propre démarche. D'autre part, être une femme dans les espaces publics à Johannesburg, c'est se trouver dans une position de vulnérabilité, qui suppose d'adopter un comportement de réserve et de prudence afin de ne pas susciter le rejet ou la convoitise. En effet, les méthodes d'enquête de terrain comme les questionnaires sont difficilement utilisables dans la rue à Johannesburg, car un homme noir accosté par une femme blanche lui demandant d'où il vient, ce qu'il fait là ou s'il aime cet endroit, soupçonnera des intentions tout autres que scientifiques.

Ainsi, sur deux des études de cas que j'ai menées dans le centre-ville de février à avril 2009, je n'ai pu réaliser des questionnaires que dans un seul cas. Ce dernier présentait deux avantages sur le plan méthodologique. Tout d'abord, l'œuvre d'art public étudiée était installée dans un quartier du centre-ville relativement mixte et étudiant, du fait de la proximité 
de l'université du Witwatersrand ${ }^{6}$. Je pouvais donc passer relativement inaperçue. Ensuite, ce lieu comprenant un arrêt de bus et de taxis collectifs, beaucoup de personnes acceptaient de répondre à mes questions pour faire passer le temps.

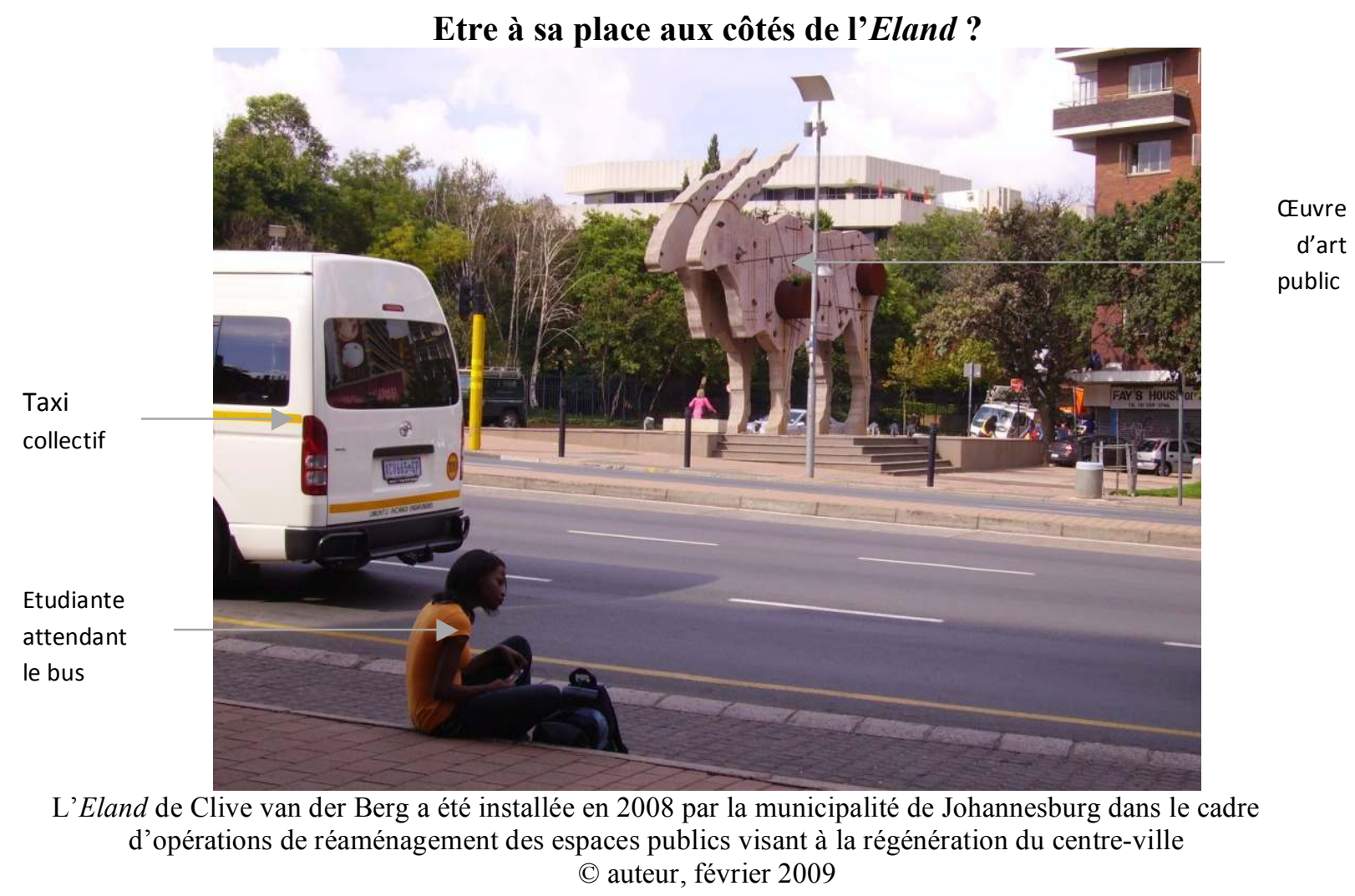

Dans ces conditions, je me sentais suffisamment acceptée dans l'espace public pour conduire mes enquêtes. J'étais « in-place » (Cresswell, 1996), en accord avec les règles tacites du lieu. L'espace était pour moi accessible physiquement et mentalement.

En revanche, lors de la deuxième étude de cas, l'œuvre d'art public examinée se trouvait dans un espace public du centre-ville fréquenté majoritairement par des hommes noirs. Dès que je m'arrêtais dans cet espace, je me sentais épiée, scrutée, dévisagée. Le poids des regards me faisait sentir que je n'avais pas à être là. La situation s'est encore compliquée quand, à partir du mois de mars, des refugiés zimbabwéens ont investi cet espace :

\footnotetext{
${ }^{6}$ L'université du Witwatersrand est l'une des deux universités de Johannesburg, et parmi les plus réputées d'Afrique du Sud. Réservée aux Blancs pendant l'apartheid, elle a été officiellement « déségréguée » dès 1990.
} 


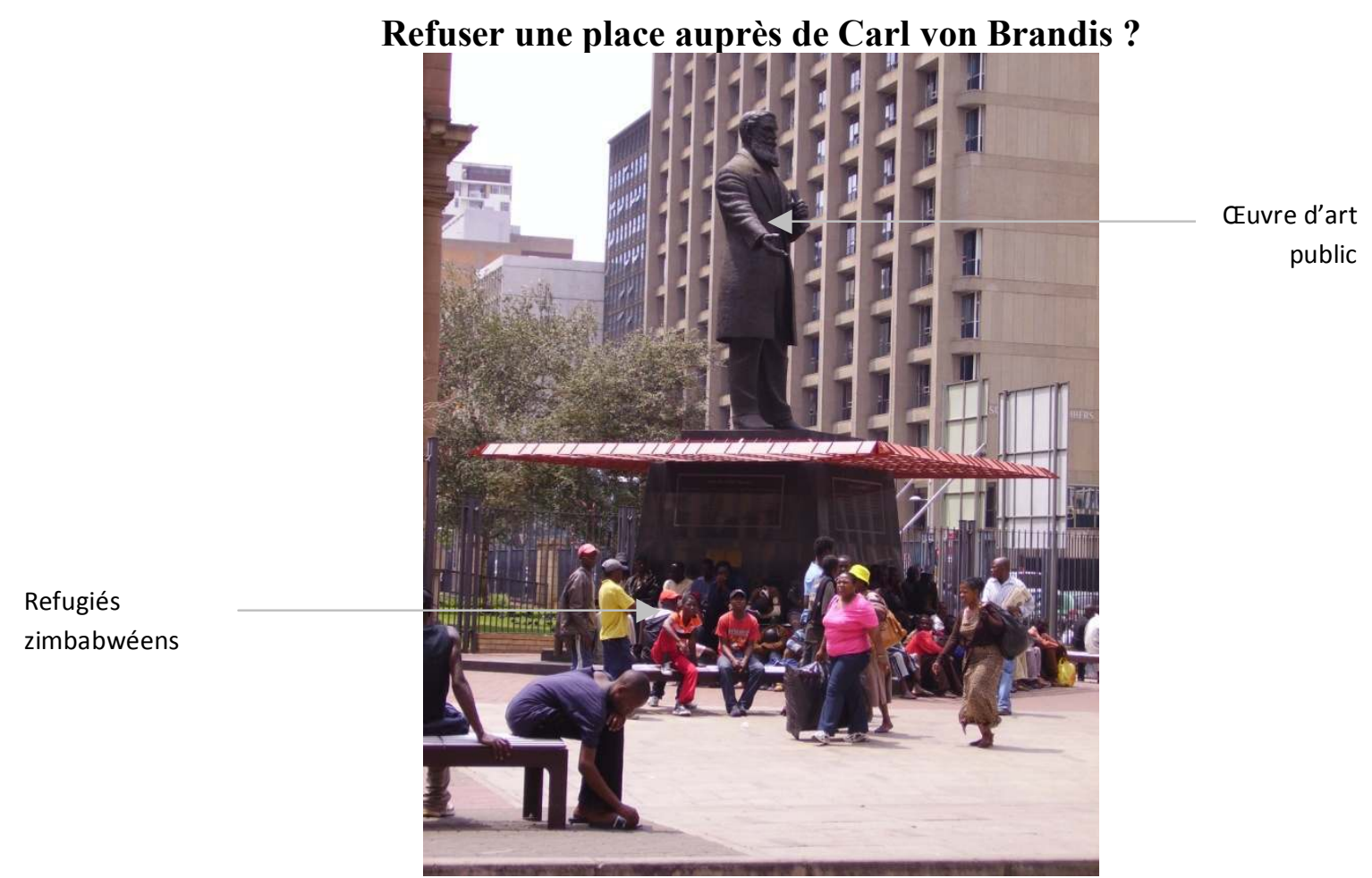

La statue de Carl von Brandis, premier magistrat de la ville de Johannesburg, a été érigée en 1965 et rénovée en 2008 à la demande de la municipalité parallèlement au réaménagement de l'espace public

(C) auteur, mars 2009

Désormais, ce n'était pas tant le regard des autres qui me rendait mal à l'aise que mon propre regard sur le sens de ma présence dans ce lieu. Comment interroger des personnes sur leurs perceptions de l'art public alors qu'elles n'ont ni toit, ni nourriture ? Dans ces conditions, il me semblait illégitime d'être là, et d'autant plus de questionner ces gens. Je trouvais cet espace mentalement, et même moralement, inaccessible en tant que chercheur travaillant sur l'art public.

Ces deux études de cas mettent donc en évidence, à travers mes propres perceptions, différents degrés en termes d'accessibilité des espaces publics. En tant que chercheuse blanche, je peux me sentir rejetée d'un espace juridiquement public soit parce que les regards des autres usagers de l'espace me font comprendre que je ne suis pas à ma place, soit parce que mon propre regard m'exclut de cet espace. Est-ce à dire que l'accessibilité des espaces juridiquement publics à Johannesburg, bien qu'effective pour tous en droit, serait en pratique fonction du sexe, de la couleur de peau ou de la classe sociale ? Si tel est le cas, on aurait alors une juxtaposition d'espaces publics hétérogènes, que l'on pourrait qualifier de communautaires en ce qu'ils seraient appropriés par des communautés sexuelles, "raciales » et/ou sociales. Mais la constitution de tels espaces communautaires, en ce qu'ils annihilent la possibilité même de rencontre avec l'autre, ne remet-elle pas en cause la notion même d'espace public (Ghorra-Gobin, 2001) ? Sur le plan méthodologique, face à l'hétérogénéité de ces espaces, comment dépasser la simple juxtaposition des études de cas pour pouvoir prétendre à un discours plus général sur les espaces publics à Johannesburg ? Ces espaces sont-ils différents au point de ne pouvoir être appréhendés à l'aune d'une même notion?

\section{Conclusion}

Parce que les espaces publics à Johannesburg sont des espaces dont l'existence même fait problème, se demander comment les étudier est une question cruciale en ce qu'elle est en 
elle-même constitutive de l'objet de recherche. La manière dont je peux ou non pratiquer ces espaces, en tant que femme blanche, est en effet déjà pour moi un moyen de les appréhender. Mes propres perceptions, peurs et préjugés me permettent de mettre à jour des formes d'exclusion qui les parcourent et qui implicitement les façonnent. En même temps, ces représentations m'invitent aussi à chercher d'autres formes d'enquête me permettant de trouver ma place en tant que chercheur travaillant sur et dans les espaces publics johannesburgeois. L'entrée par l'art pourrait-elle offrir un nouveau regard pour comprendre les espaces publics? L'art public serait-il un moyen de contrer la différenciation progressive des espaces publics, ou contribuerait-il, à l'inverse, à leur communautarisation ?

\section{Bibliographie}

Beavon K., 2004, Johannesburg: The Making and Shaping of the City, Pretoria, UNISA Press, 374 p.

Boisteau C., 2003, Sécurité, dynamiques urbaines et privatisation de l'espace à Johannesburg, mémoire de DEA, Genève, Institut Universitaire d'Etudes du Développement, 98 p.

Capron G., 1998, «Les centres commerciaux à Buenos Aires : les nouveaux espaces publics de la ville de la fin du XXe siècle », Les annales de la recherche urbaine, ${ }^{\circ} 78$, pp. 55-63.

Creswell T., 1996, In Place/Out of Place: Geography, Ideology and Transgression, Minneapolis, University of Minnesota Press, $201 \mathrm{p}$.

Ghorra-Gobin C. (dir.), 2001, Réinventer le sens de la ville. Les espaces publics à l'heure globale, Paris, L'Harmattan, 266 p.

Guillaume P., 2001, Johannesburg : Géographies de l'exclusion, Paris, Karthala, 391 p.

Houssay-Holzschuch M., Teppo A., 2009, "A mall for all? Race and public space in post-apartheid Cape Town”, in Cultural Geographies, n¹6, pp. 351-379.

Mabin A., 2001, "Past Formation, Present Recomposition and Future Significance of Public Space(s) in South African Cities", in Ghorra-Gobin C. (dir.), Réinventer le sens de la ville. Les espaces publics à l'heure globale, Paris, L'Harmattan, pp. 245-258.

Mitchell D., 2005, "The S.U.V. model of citizenship: floating bubbles, buffer zones, and the rise of the "purely atomic" individual", in Political Geography, n²4, pp. 77-100.

Paquot T., 2009, L'espace public, Paris, La Découverte, 125 p. 